\title{
HISTÓRIA E VARIAÇÃO \\ MORFOSSINTÁTICA ENTRE O \\ QUIMBUNDO E O PORTUGUÊS NA \\ REGIÃO DE KWANZA NORTE, EM \\ ANGOLA: FORMAÇÃO DO PLURAL, CONCORDÂNCIA DE GÊNERO E FORMAÇÃO DO DIMINUTIVO E DO AUMENTATIVO DOS SUBSTANTIVOS
}

HISTORIA Y VARIACIÓN MORFOSINTÁCTICA ENTRE KIMBUNDU Y PORTUGUÉS EN LA REGIÓN DE KWANZA NORTE, EN ANGOLA: FORMACIÓN DEL PLURAL, CONCORDANCIA DE GÉNERO Y FORMACIÓN DEL DIMINUTIVO Y DEL AUMENTATIVO DE LOS SUSTANTIVOS

HISTORY AND MORPHOSYNTACTIC VARIATION BETWEEN KIMBUNDU AND PORTUGUESE IN ANGOLA'S KWANZA NORTH PROVINCE: PLURAL FORMATION, GENDER AGREEMENT, AND AUGMENTATIVE AND DIMINUTIVE NOUN FORMATION

João Lourenço António* Universidade da Beira Interior

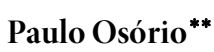
Universidade da Beira Interior

RESUMO: A pesquisa aqui apresentada baseia-se no estudo da convivência entre duas línguas - quimbundo e português - no município de Cazengo-Ndalatando, uma província da região Kwanza Norte, em Angola. Uma observação sociolinguística do contato e uso simultâneo entre as duas línguas mostra claramente situações de interferência do quimbundo na língua portuguesa

\footnotetext{
*Mestreem Estudos Lusófonos pela Universidadeda Beira Interior (Covilhã, Portugal). E-mail: <twaxinga@outlook.pt>. 
(língua oficial em Angola), o que é possível comprovar pela análise linguística de enunciados orais recolhidos diretamente na zona geográfica em questão e cujo juízo de aceitação linguística foi atestado por um dos autores deste trabalho, que é falante nativo de quimbundo. Neste artigo são, assim, analisados três fenômenos linguísticos de interferência do quimbundo no português, indicadores de variação morfossintática nos registros orais da língua portuguesa: formação do plural, concordância de gênero, além da formação do diminutivo e do aumentativo dos substantivos.

PALAVRAS-CHAVE: Interferência. Plural. Concordância. Diminutivo. Aumentativo.

RESUMEN: Esta investigación se basa en el estudio de la convivencia entre dos lenguas - kimbundu y portugués - en el municipio de Cazengo-Ndalatando, una provincia de Kwanza Norte, en Angola. Una observación sociolingüística del contacto y uso simultáneo entre las dos lenguas mencionadas muestra claramente situaciones de interferencia del kimbundu en la lengua portuguesa (lengua oficial en Angola), circunstancia que se verifica a través del análisis lingüístico de enunciados orales recogidos directamente en la zona geográfica referida y cuyo juicio de aceptación lingüística ha sido certificado por uno de los autores de este trabajo, que es hablante nativo de kimbundu. En este artículo se analizan tres fenómenos lingüísticos de interferencia del kimbundu en portugués, indicadores de variación morfosintáctica en los registros orales de la lengua portuguesa: la formación del plural, la concordancia de género y la formación del diminutivo y del aumentativo de los sustantivos.

PALABRAS CLAVE: Interferencia. Plural. Concordancia. Diminutivo. Aumentativo.

ABSTRACT: This research is based on the study of the coexistence of two languages - Kimbundu and Portuguese - in Angola's Northern Kwanza region of Cazengo-Ndalatando. A sociolinguistic observation of the contact and simultaneous use of these languages clearly denotes cases of Kimbundu interference in Portuguese (Angola's official language). This observation is supported by the linguistic analysis of oral utterances directly collected at the aforementioned geographical area, and whose linguistic acceptance judgment was confirmed by one of the authors, a native speaker of Kimbundu. In this paper, we analyze three cases of Kimbundu interference in Portuguese, which indicate morphosyntactic variation in the oral records of Portuguese: plural formation, gender concordance, and diminutive and augmentative noun formation.

KEYWORDS: Interference. Plural. Agreement. Diminutive. Augmentative.

\section{INTRODUÇÃO}

Kwanza Norte é uma das dezoito províncias de Angola, cujas fronteiras são, ao norte, as províncias de Uíge e Bengo; ao sul, a província de Kwanza Sul; a leste, a província de Malanje e, a oeste, a província de Luanda. Com dez municípios, localizada no interior noroeste de Angola, Kwanza Norte conhece uma altura que varia entre aproximadamente $500 \mathrm{~m}$ a $1500 \mathrm{~m}$ em relação ao nível do mar.

Os habitantes de Kwanza Norte são majoritariamente quimbundos (isto é, pertencem à etnia ambundo, grupo etnolinguístico autóctone). A população de Kwanza Norte pertence à família linguística banto e autodenomina-se grupo etnolinguístico ambundo; de acordo com os saberes difundidos oralmente entre alguns anciães, a designação ambundo refere-se aos súditos do antigo reino do Ndongo. Tal como indicado por Mingas (2000, p. 32), “o quimbundo é a língua nacional (...), língua oficial do país, fruto da colonização implementada por Portugal a esta parcela da África Austral”.

Ndalatando é a capital do município do Cazengo e também a sede da província de Kwanza Norte. Por sua arquitetura, mostra-se como um cartão postal da antiga administração colonial portuguesa. Primeiramente chamada "Vila Salazar", nome que provém do ex-dirigente colonial português, António de Oliveira Salazar, passou a chamar-se Ndalatando no período pós-independência de Angola; nessa época, a cidade era povoada essencialmente por colonos portugueses, que a tinham construído e povoado. Ndatalando é, então, uma cidade na qual a língua portuguesa constituiu-se como língua de uso coloquial pela comunidade local. Nos arredores da vila, viviam comunidades nativas, alguns elementos das quais conheciam (ainda que parcamente) o português, porque serviam de mão de obra nas casas dos senhores colonos. A restante população desconhecia totalmente a língua portuguesa, dominando unicamente o quimbundo, a língua local. Desta aproximação entre as duas línguas e os dois povos, surgiram fenômenos de bilinguismo, de diglossia e de interferências do quimbundo no português.

António \& Osório | História e variação morfossintática entre o quimbundo e o português na região de Kwanza Norte... 
No decurso de diferentes momentos da sua história de contato com as frentes de colonização, parte da população conheceu certa mestiçagem com os colonos, o que provocou uma situação de diglossia. Ainda hoje, nas áreas um pouco mais distantes de Ndalatando, algumas crianças, mulheres e idosos falam unicamente a língua quimbundo. No entanto, ocorrem também casos em que o quimbundo passa por uma situação de desuso, com domínio quase exclusivo da língua portuguesa por parte de crianças, jovens e pessoas de mais idade. Todavia, "para muitos dos colonizados, o bom conhecimento da língua portuguesa era a condição de destaque para quem quisesse aceder a qualquer posição na sociedade colonial" (MINGAS, 2000, p. 32).

Do mesmo modo, atualmente, alguns habitantes de Cazengo pensam que dominar a língua portuguesa e não falar a sua língua de origem granjeia-lhes destaque e boa posição social, apesar de uma parte dos cazenguenses ter nascido no seio de famílias cuja língua materna é o quimbundo. Ainda que muitos prefiram o uso do português em detrimento do quimbundo, este estará sempre presente no seio da comunidade, por intermédio de cerimônias tradicionais (casamentos, funerais, atos religiosos diversos), assim como nos festivais tradicionais, tanto na zona urbana como na rural.

Apesar da questão de identidade e da consciência nacional que a comunidade vem ganhando acerca da importância da sua cultura, a verdade é que o quimbundo conhece uma situação de desvantagem face à língua portuguesa, como se pode constatar na política de inserção da língua quimbundo, em fase experimental no sistema educativo nacional e que vigora apenas em algumas escolas do município.

Do ponto de vista sociolinguístico, no que concerne aos falantes que possuem o quimbundo como língua materna e o português como língua segunda, no município de Cazengo, destacam-se as interferências lexicais e morfológicas, com maiores incidências no diminutivo e no aumentativo dos substantivos, na concordância de gênero e na inversão do plural; há ainda várias outras realizações, que apresentam menor expressividade na comunidade linguística em questão. Por exemplo, a palavra kambuta é um empréstimo do quimbundo usado na região em lugar de "baixinho":

(1) O marido dela é kambuta.

(1') O marido dela é baixinho.

(2) O meu kandenge está em casa.

(2’) O meu irmãozinho está em casa.

Vindos do quimbundo, os termos acima são empréstimos lexicais usados no município de Cazengo e em quase toda a extensão da área geolinguística do território angolano. Nestes casos de interferência fica bem claro que o ponto máximo dessa variação ocorre sobretudo na comunicação oral. Nota-se, nesse caso, uma adaptação do léxico quimbundo disponível no repertório linguístico dos falantes, diante de uma indisponibilidade ou estranheza de certas palavras da língua portuguesa.

De fato, a interferência, enquanto fenômeno linguístico resultante do contato direto entre línguas, é uma consequência de diversos fatores externos: políticos, econômicos, sociais, culturais e psicológicos, com repercussões que levam a adaptações, integrações e reestruturações linguísticas inevitáveis, tanto do ponto de vista diacrônico quanto do sincrônico (ALMEIDA, 2001, p. 24).

\section{BREVE CARACTERIZAÇÃO DE ALGUMAS ESTRUTURAS DA LÍNGUA QUIMBUNDO}

Certos critérios estruturais do quimbundo foram definidos de forma clara por Ngunga (2012) e Sassoma (2015). Por exemplo, os indicadores de gênero são prefixos por meio dos quais os nomes se distribuem em classes (1 a 19), sendo que as classes, por sua vez, se encontram associadas em pares que são "singular" e "plural" (de um gênero). Há, todavia, casos na língua quimbundo de nomes que representam o singular, cujo prefixo é o plural de outra classe. Esses nomes realizam o seu plural com a classe 10. Também encontramos prefixos isolados, somente no singular, sem uma classe par. O plural de tais prefixos pertence às classes 6 e 10 . Vejamos alguns exemplos: 
(3) mako (singular): "mão" (classe 6)

(3’) jimako (plural): "mãos" (classe 10)

(4) lumwenu (singular): "espelho” (classe 11)

(4') malumwenu (plural): "espelhos" (classe 6)

(5) uhaxi (singular): "doença”" (classe 14)

(5’) mawuhaxi (plural): "doenças" (classe 6)

Para a formação da frase, quando uma palavra tem um prefixo nominal (PN) indicador de classe como prefixo inicial, toda palavra subordinada deve concordar com o PN. O prefixo inicial presente nas palavras subordinadas denomina-se "prefixo de concordância" (PD).

A língua não possui uma categoria semântica claramente definida para indicar o gênero: os prefixos nominais regem o funcionamento do número, tanto para o singular, quanto para o plural. Convém observar, no entanto, que o número de classificadores da língua quimbundo varia de acordo com cada investigador. Segundo Peres (2006), o quimbundo contém 18 prefixos ou classes de palavras: 14 nominais, um verbal e três locativos, podendo-se esquematizar os classificadores ou prefixos nominais do seguinte modo:

\begin{tabular}{|c|c|c|c|}
\hline \multicolumn{2}{|c|}{ Prefixos nominais ou classificadores do Quimbundo } & \multicolumn{2}{|c|}{ Exemplos } \\
\hline & & Quimbundo & Português \\
\hline 1- singular & $\mathrm{Mu}$ & mukalakadi & trabalhador \\
\hline 2-plural & A & akalakadi & trabalhadores \\
\hline 3- singular & $\mathrm{Mu}$ & mulundu & montanha \\
\hline 4- plural & $\mathrm{mi}$ & milundu & montanhas \\
\hline 5- singular & $\mathrm{di}$ & dikanda & pata \\
\hline 6-plural & $\mathrm{ma}$ & makanda & patas \\
\hline 7- singular & $\mathrm{ki}$ & kituxi & pecado \\
\hline 8-plural & $\mathrm{i}$ & ituxi & pecados \\
\hline 9- singular & $\mathrm{f}, \mathrm{h}, \mathrm{i}, \mathrm{k}, \mathrm{m}, \mathrm{n}, \mathrm{o}, \mathrm{ph}, \mathrm{s}, \mathrm{t}, \mathrm{x}, \mathrm{z}$ & ndungu & pimenta \\
\hline 10- plural & ji & jindungu & pimentas \\
\hline 11- singular & lu & lwoso & arroz \\
\hline 12- singular & $\mathrm{ka}$ & kandenge & pequenino/criancinha \\
\hline 13-plural & tu & tundenge & pequeninos/criancinhas \\
\hline 14- singular & $\mathrm{u}$ & ulaji & maluquice \\
\hline
\end{tabular}




\begin{tabular}{c|c|c|c} 
15- ifinitivo & $\mathrm{ku}$ & kumatuka & bhobha \\
\hline 16 - locativo & $\mathrm{bh}$ & kunaa & ali \\
\hline 17 - locativo & $\mathrm{ku}$ & mu nzo dya kusaka & dentro do hospital
\end{tabular}

Tabela 1: Prefixos nominais ou classificadores da língua quimbundo

Fonte: produzida pelos autores

Também de acordo com Sassoma (2015), a formação do plural na língua quimbundo se faz por meio da prefixação. Assim, segundo o autor, os substantivos agrupam-se num certo número de morfemas que se colocam no seu início para indicar o número, singular ou plural: esses morfemas são por ele denominados "prefixos nominais" ou "classificadores". Segundo Peres (2006), os prefixos nominais são os catalisadores em todas as línguas do grupo banto e estão presentes em todas as circunstâncias. No quimbundo, os prefixos nominais regem o funcionamento, das frases, pois regem as relações de concordância. A tabela seguinte ilustra as formas de construção do plural:

\begin{tabular}{|c|c|c|c|c|c|}
\hline \multirow{2}{*}{\multicolumn{2}{|c|}{ Classes e marcas do singular }} & \multirow{2}{*}{\multicolumn{2}{|c|}{$\begin{array}{l}\text { Classes correspondentes e } \\
\text { marcas do plural }\end{array}$}} & \multicolumn{2}{|c|}{ Exemplos } \\
\hline & & & & Singular & Plural \\
\hline 1 & $\mathrm{mu}$ & 2 & $\mathrm{a}$ & mulongexi (professor) & alongexi (professores) \\
\hline 3 & $\mathrm{mu}$ & 4 & $\mathrm{mi}$ & mulonde (ponte) & milonde (pontes) \\
\hline 5 & di & 6 & ma & dikanu (boca) & makanu (bocas) \\
\hline 7 & ki & 8 & $\mathrm{i}$ & kyezo (vassoura) & isoneku (vassouras) \\
\hline 9 & $\begin{array}{c}\mathrm{f}, \mathrm{h}, \mathrm{i}, \mathrm{k}, \mathrm{m}, \mathrm{n}, \mathrm{o}, \mathrm{ph}, \mathrm{s}, \mathrm{t}, \\
\mathrm{x}, \mathrm{z}\end{array}$ & 10 & ji & fundanga (pólvora) & jifundanga (pólvoras) \\
\hline 11 & lu & 10 e 6 & $\mathrm{ji} / \mathrm{ma}$ & lukwako (braço) & malukwako (braços) \\
\hline 12 & ka & 13 & tu & kanjila (passarinho) & tunjila (passarinhos) \\
\hline 14 & $\mathrm{u}$ & 10 e 6 & $\mathrm{ji} / \mathrm{ma}$ & ufunu (profissão) & jifunu (profissões) \\
\hline
\end{tabular}

Tabela 2: Formação do plural em quimbundo

Fonte: produzida pelos autores 


\section{INTERFERÊNCIAS DO QUIMBUNDO NO PORTUGUÊS}

\subsection{FORMAÇÃO DO PLURAL}

Em português, o plural é formado por sufixação, por norma, pela adição do grafema $<\mathrm{s}>$ no final de uma palavra na sua forma singular, principalmente quando esta se termina por uma vogal. Segundo Raposo (2013), praticamente todos os nomes do português admitem, do ponto de vista estritamente morfológico, duas versões, uma singular e outra plural, com exceção dos nomes paroxítonos terminados $\mathrm{em}<\mathrm{s}>$, que são morfologicamente invariáveis em número.

Como vimos, em quimbundo os substantivos agrupam-se num certo número de morfemas que se colocam no seu início para indicar se se trata de singular ou plural: são os prefixos nominais ou classificadores. Por esta razão, os falantes de quimbundo que têm o português como L2 tendem a aproximar as duas línguas, pela introdução das regras do quimbundo. Vejamos:

(6) Ele tem muitos carros (Português padrão)

(6’) Ele tem muito scarro (Português de Kwanza Norte)

(7) Temos muitas casas (Português padrão)

(7’) Temos muita scasa (Português de Kwanza Norte)

O tratamento do sufixo $<$ s $>$ do português como um prefixo fica evidente nesses exemplos, ou seja, as regras do quimbundo são transpostas para o português. Pode ser interessante comparar palavras isoladas das duas línguas:
(8) dikalu / carro
(8') makalu / carros
(9) nzo / casa
(9') jinzo / casas
(10) kinama / perna
(10') inama / pernas
(11) mutu / pessoa
(11') atu/pessoas

Como se vê, o mesmo fenômeno - a adjunção de um PN marcador de singular ou de plural a um tema nominal (TN) - ocorre no processo morfológico aqui descrito. Em (8) e (8'), temos que o morfema di é um PN de classe 5, kalu sendo o TN: para realizar o plural, junta-se o PN de classe 6 “ma” ao TN “kalu”. Em (9) e (9'), n é um PN de classe 9, enquanto nzo é o TN. O plural é formado pela adjunção de ji, PN de classe 10 ao TN. Em (10) e (10’), nama é o TN, enquanto ki é o PN singular (de classe 7) e i o PN plural de classe 8. Do mesmo modo, em (11) e (11'), tu é o TN ao qual a adjunção do PN mu forma o singular e a adjunção do PN a forma o plural.

\subsection{CONCORDÂNCIA DE GÊNERO}

No que diz respeito à concordância de gênero, o quimbundo não procede como o português, como se poderia esperar. Por exemplo, os termos, Mona (singular) e Ana (plural), em quimbundo significam, respectivamente, "filho/a" ou "filhos/as". Alguns falantes do 
português de Ndalatando, têm "quimbundizado" o português com expressões como "filho de homem" e "filha de mulher", para "filho" e "filha", respectivamente, em casos em que é necessário elucidar o gênero da pessoa referida. Também é possível acrescentar os substantivos diyala ("homem") e muhatu ("mulher") ao substantivo mona. Comparem-se

(12) mona diyala

[filho/a + homem] = filho de homem

(12') mona wa diyala $(\mathbf{m o}+\mathrm{na}+\mathbf{w a}+\mathrm{di}+$ yala $)$

$[\mathrm{PN} 1+\mathrm{TN}+\mathrm{PC}+\mathrm{PN} 5+\mathrm{TN}]$

(13) ana mayala

[filhos/as + homens] = filhos de homens

(13') ana a mayala $(\mathbf{a}+\mathbf{n a}+\mathbf{a}+\mathbf{m a}+\mathbf{y a l a})$

$[\mathrm{PN} 2+\mathrm{TN}+\mathrm{PC}+\mathrm{PN} 6+\mathrm{TN}]$

(14) mona muhatu

$[$ filha + mulher $]=$ filha de mulher

(14) mona wa muhatu (mo+na+wa+mu+hatu)

$[\mathrm{PN} 1+\mathrm{TN}+\mathrm{PC}+\mathrm{PN} 1+\mathrm{TN}]$

(15) ana ahatu

[filhas + mulher $]=$ filhas de mulheres

(15) ana ahatu (a+na+a+ma+ahetu)

$[\mathrm{PN} 2+\mathrm{TN}+\mathrm{PC}+\mathrm{PN} 5+\mathrm{TN}]$

(16) mona ndenge

$[$ filho/a + pequeno/a $]=$ filho/a pequeno/a

(16) mona wa ndenge (mo+na+wa+ $(\mathbf{n})+$ ndenge $)$

$[\mathrm{PN} 1+\mathrm{TN}+\mathrm{PC}+\mathrm{PN} 9+\mathrm{TN}]$

(17) ana ndenge

[filho/as + pequeno/as] = filho/as pequeno/as

$\left(17^{\prime}\right)$

ana a ndenge $(\mathbf{a}+\mathbf{n a}+\mathbf{a}+\emptyset(\mathbf{n})+$ ndenge $)$

$[\mathrm{PN} 2+\mathrm{TN}+\mathrm{PC}+\mathrm{PN} 9+\mathrm{TN}]$

Esses exemplos ajudam-nos a perceber claramente algumas interferências que se registram no português falado por uma franja dos habitantes de Cazengo, em Ndalatando. Para Mingas (2000, p. 68-83), ao contrário do que acontece para o português, o quimbundo conhece diversas classes de substantivos representadas por prefixos, que se organizam em pares singular/plural, mas para os quais as distinções de gênero não são importantes. A autora reforça que, ao identificar a inexistência da distinção de gênero em quimbundo, os falantes podem apresentar uma incapacidade para estabelecer na língua portuguesa a concordância do determinante com o substantivo, como nos mostram exemplos como "meu casa", "minha carro", "minha parente" (por "meu parente").

Nota-se, em quimbundo, uma oposição entre o singular e o plural dos substantivos, enquanto em português, além dessa oposição, verifica-se também a oposição entre masculino e feminino (MINGAS, 2000, p. 68): em quimbundo, o substantivo mona é biforme, ou seja, não define o gênero. A definição do gênero se faz, muitas vezes, pela adjunção de outros substantivos. Estas e outras questões estão na base das interferências que focamos neste estudo. 
O diminutivo em português se expressa pelo uso de determinadas estruturas morfológicas que se acrescentam à parte final da palavra (com ou sem modificação desta), dando origem a uma nova palavra, a qual, quando comparada com a primeira, adquire um significado que apresenta uma 'ideia' de diminuição, cuja referência pode ser o espaço físico (altura ou largura), uma qualidade ou característica, uma quantidade etc.

\begin{tabular}{|c|c|c|c|}
\hline Sufixo & Exemplificação & Sufixo & Exemplificação \\
\hline - inho, -a & toquinho, vozinha & -elho, -a & folhelho, rapazelho \\
\hline -zinho, -a & cãozinho & -ejo & animalejo, lugarejo \\
\hline -ino, -a & pequenino, cravina & -ilho, -a & pecadilho, tropilha \\
\hline$-i m,-a$ & espadim, fortim & -ete & artiguete, lembrete \\
\hline -acho, -a & fogacho, riacho & -eto, -a & esboceto, saleta \\
\hline -icho, -a & governicho, barbicha & -ito, -a & rapazito, casita \\
\hline -ucho, -a & papelucho, casucha & -zito, -a & jardinzito, florzita \\
\hline -ebre & casebre & -ote, -a & velhote, velhota \\
\hline$-e c o,-a$ & livreco, soneca & -isco, -a & chuvisco, talisca \\
\hline$-\mathrm{ico},-\mathrm{a}$ & burrico, marica(s) & -usco, -a & chamusco, velhusco \\
\hline -ela & ruela, viela & -ola & fazendola, rapazola \\
\hline
\end{tabular}

Tabela 3: Principais sufixos diminutivos portugueses (SANTANA, 2017, p. 43)

Fonte: produzida pelos autores

Como se poderia esperar, as regras de formação do diminutivo em português são muito diferentes das regras de formação do diminutivo em quimbundo, língua na qual o diminutivo se forma por prefixação, como nos ilustra a Tabela seguinte:

\begin{tabular}{c|c|c|c}
\multirow{2}{*}{$\begin{array}{c}\text { Substantivos em } \\
\text { quimbundo }\end{array}$} & Português & Singular & Plural \\
\cline { 2 - 4 } & casa - casinha - casinhas & kanzo & tunzo \\
\hline inzo & pedra - pedrinha - pedrinhas & kaditadi & tuditadi
\end{tabular}




\begin{tabular}{|c|c|c|c|}
\hline imbwa & cão - cãozinho - cãozinhos & kambwa & tumbwa \\
\hline imbya & panela - panelinha - panelinhas & kambya & tumbya \\
\hline mutwe & cabeça - cabecinha-cabecinhas & kamutwe & tumutwe \\
\hline xingo & pescoço - pescocinho - pescocinhos & kaxingo & tuxingo \\
\hline mulembu & dedo - dedinho - dedinhos & kamulembu & tumilembu \\
\hline menya & água - aguinha - aguinhas & kamenya & tumenya \\
\hline njila & pássaro - passarinho - passarinhos & kamwimbo & tumimbu \\
\hline dikalu & carro - carrinho - carrinhos & kadikalu & tumakalu \\
\hline mbiji & peixe - peixinho - peixinhos & kambiji & tumbiji \\
\hline xito & carne - carnezinha - carnezinhas & kaxitu & tuxitu \\
\hline wabuta & baixo - baixinho - baixinhos & kabuta & tubuta \\
\hline walebha & alto - altinho - altinhos & kalebha & tulebha \\
\hline ngiji & rio - riacho - riachos & kangiji & tungiji \\
\hline dibubu & mudo - mudinho - mudinhos & kabubu & tububu \\
\hline muxilu & surdo - surdinho - surdinhos & kamuxilu & tumixilu \\
\hline kifofo & cego - ceguinho - ceguinhos & kafofo & tufofo \\
\hline
\end{tabular}

Tabela 4: Formação do diminutivo em quimbundo

Fonte: produzida pelos autores

Em quimbundo, o diminutivo é formado por prefixação. Trata-se de dois prefixos nominais: ka, de classe 12, para o singular, e tu, de classe 13, para o plural. Uma parte significativa da comunidade de Cazengo transpõe esse processo de prefixação para o português: são interferências de ordem lexical, como já observado por Mingas (2000) e como ilustram os exemplos abaixo:

(18) Está contente porque comprou um kacarro.

(18') Está contente porque comprou um carrinho.

(19) Trouxe tupeixe para o jantar.

(19') Trouxe peixinhos para o jantar.

(20) Esta é a nossa kacidade.

(20’) Esta é a nossa cidadezinha.

(21) As tucasa. 
Contrariamente ao que indica Mingas (2000), não se trata unicamente de um uso isolado entre indivíduos analfabetos, pois essas construções também são encontradas entre indivíduos escolarizados. De acordo com Sassoma (2015), trata-se de uma interferência de ordem morfológica, devida à coabitação entre duas línguas com estruturas completamente diferentes. Passemos, agora, a uma análise da estrutura dessas mesmas palavras em quimbundo:

(22) $\mathbf{k a}+\mathbf{d i}+\mathbf{k a l u}=\mathbf{k a d i k a l u}$ ("carrinho”)

$[\mathrm{PA} 12+\mathrm{PN} 5+\mathrm{TN}]$

(22') $\quad \mathbf{t u}+\mathbf{m a}+\mathbf{k a l u}=\mathbf{t u d i k a l u}($ “carrinhos")

$[\mathrm{PA} 13+\mathrm{PN} 5+\mathrm{TN}]$

(23') $\mathbf{k a}+\emptyset(\mathbf{m})+\mathbf{m b i j i}=\mathbf{k a m b i j i}($ ("peixinho”)

$[\mathrm{PA} 12+\mathrm{PN} 9+\mathrm{TN}]$

(23') $\quad \mathbf{t u}+\emptyset(\mathbf{m})+\mathbf{m b i j i}=\mathbf{t u m b i j i}$ (“peixinhos”)

$[\mathrm{PA} 13+\mathrm{PN} 9+\mathrm{TN}]$

(24) $\mathbf{k a}+\emptyset(\mathbf{m})+\mathbf{m b a n z a}=\mathbf{k a m b a n z a}$ (“cidadezinha”)

$[\mathrm{PA} 12+\mathrm{PN} 9+\mathrm{TN}]$

(24') $\quad \mathbf{t u}+\emptyset(\mathbf{m})+\mathbf{m b a n z a}=\mathbf{t u m b a n z a}$ (“cidadezinhas”)

$[\mathrm{PA} 13+\mathrm{PN} 9+\mathrm{TN}]$

(25) $\mathbf{k a}+\mathbf{i}+\mathbf{n z o}=\mathbf{k a i n z o}($ casinha $)$

$[\mathrm{PA} 12+\mathrm{PN} 9+\mathrm{TN}]$

(25) $\quad \mathbf{t u}+\mathbf{i}+\mathbf{n z o}=\mathbf{t w i n z o}$ (casinhas)

$[\mathrm{PA} 13+\mathrm{PN} 9+\mathrm{TN}]$

Voltemo-nos, agora, para o aumentativo. Em quimbundo o aumentativo é formado pela anteposição do prefixo ki, que pertence à classe 7. Vejamos alguns exemplos:

\begin{tabular}{c|c|c}
\multirow{2}{*}{ Substantivos em quimbundo } & & Exemplos de aumentativo \\
\cline { 2 - 3 } & Quimbundo & Português \\
\hline muhatu & kimuhatu & mulherona, grande, mulher de respeito \\
\hline diyala & kidiyala & "homenzão", de respeito, homem grande \\
\hline imbwa & kimbwa & cão forte, de grande estatura. \\
\hline dikanda & kidikanda & patão \\
\hline mutwe & kimutwe & cabeçudo
\end{tabular}




\begin{tabular}{|c|c|c|}
\hline nzebu & kinzebu & babão \\
\hline dilonga & kidilonga & pratão \\
\hline mazo & kimazo & dentola \\
\hline mwezo & kimwezo & barbudo \\
\hline dikalu & kidikalau & carrão \\
\hline ndemba & kindemba & cabeludo \\
\hline dilaji & kidilaji & grande maluco \\
\hline ditaku & kiditaku & rabão \\
\hline dyeso & kidyeso & olhos grande, olhudo. \\
\hline kujiza & kijiza & teimoso \\
\hline dileku & kileku & sabichão \\
\hline dimi & kidime & linguarudo \\
\hline
\end{tabular}

Tabela 5: Formação do aumentativo em quimbundo

Fonte: produzida pelos autores

Como observamos para o diminutivo, o aumentativo também é fonte de interferências no português falado em Cazengo, inclusive entre indivíduos escolarizados. Apresentamos, abaixo, alguns exemplos:

(26) Já viste o kicarro do comandante?
(26) Já viste o carrão do comandante?

(27) O ladrão tinha uma kipata que parecia calçar 50.

(27') O ladrão tinha um patão que parecia calçar 50.

(28) Tinha aspecto de um kitio

(28') Tinha aspecto de um tiozão.

(29) Olha kicabeça dele.

(29’) Ele era cabeçudo.

(30) Tinha uma kibarba.

(30’) Era barbudo

(31) Os meus pais querem construir uma kicasa no bairro da Kipata.

(31') Os meus pais querem construir um casarão no bairro do Patão.

Na tabela abaixo, apresentamos as correspondências, para essas palavras, entre o quimbundo, o português de Cazengo e o português padrão: 


\begin{tabular}{|c|c|c|}
\hline Quimbundo & Cazengo & Português \\
\hline $\begin{array}{l}\text { kidikalu }[\mathrm{ki}+\mathrm{di}+\mathrm{kalu}] \\
\quad[\mathrm{PA} 7+\mathrm{PN} 5+\mathrm{TN}]\end{array}$ & kicarro & Carrão \\
\hline $\begin{array}{r}\text { kidikanda }[\mathrm{ki}+\mathrm{di}+\mathrm{kanda}] \\
{[\mathrm{PA} 7+\mathrm{PN} 5+\mathrm{TN}]}\end{array}$ & kipata & Patão \\
\hline $\begin{array}{r}\text { kidilemba }[\mathrm{ki}+\mathrm{di}+\mathrm{lemba}] \\
{[\mathrm{PA} 7+\mathrm{PN} 5+\mathrm{TN}]}\end{array}$ & kitio & Senhor de má aparência. \\
\hline $\begin{array}{l}\text { kimutwe }[\mathrm{ki}+\mathrm{mu}+\mathrm{twe}] \\
\quad[\mathrm{PA} 7+\mathrm{PN} 3+\mathrm{TN}]\end{array}$ & kicabeça & cabeçudo \\
\hline $\begin{array}{l}\text { kimwezo }[\mathrm{ki}+\mathrm{mu}+\mathrm{ezo}] \\
{\left[\begin{array}{l}\mathrm{PA} 7+\mathrm{PN} 3+\mathrm{TN}]\end{array}\right.}\end{array}$ & kibarba & barbudo \\
\hline $\begin{array}{l}\text { kinzo }[\mathrm{ki}+\mathrm{i}+\mathrm{nzo}] \\
\quad[\mathrm{PA} 7+\mathrm{PN} 9+\mathrm{TN}]\end{array}$ & kicasa & casarão \\
\hline $\begin{array}{r}\text { Kidikanda }[\mathrm{ki}+\mathrm{di}+\mathrm{kanda}] \\
{[\mathrm{PA} 7+\mathrm{PN} 5+\mathrm{TN}]}\end{array}$ & kipata & Patão \\
\hline
\end{tabular}

Tabela 6: Aumentativo em Quimbundo, em Português de Cazengo e em Português padrão

Fonte: produzida pelos autores

Quando utilizado como aumentativo, o classificador ki, de classe 7, pode apresentar sentidos denotativos como as ideias de grandeza, garbosidade, maleficência, entre outras. Vejamos:

\section{(32) Este é kipessoa.}

(32’) Esta é uma grande pessoa.

(33) Este é kidiabo.

(33’) Esta é uma má pessoa.

(34) Era uma kipessoa.

(34’) Era um homem forte/ uma grande pessoa/ uma má pessoa.

Este é um uso muito característico de Cazengo para atribuir qualidades a um indivíduo de acordo com a intenção expressiva do sujeito falante, sendo muito frequente nos mercados informais, aglomerados, algazarras, caminhadas nos campos agrícolas, agrupamentos após os cultos religiosos, cerimônias tradicionais.

A comparação dessas três possibilidades de expressão do aumentativo traz-nos informações interessantes: 


\begin{tabular}{|c|c|c|}
\hline Quimbundo & Português & Cazengo \\
\hline $\begin{array}{l}\text { kimutu }[\mathbf{k i}+\mathbf{m u} \mathbf{u}+\mathbf{t u}] \\
\qquad[\mathrm{PA} 7+\mathrm{PN} 1+\mathrm{TN}]\end{array}$ & grande pessoa & kipessoa \\
\hline Mwene kimutu & Ele é uma grande pessoa & \\
\hline $\begin{array}{r}\text { kikadyaphemba }[\underline{\mathrm{ki}+\mathrm{ka}+\mathrm{dyaphemba}]} \\
{[\mathrm{PA} 7+\mathrm{PN} 12+\mathrm{TN}]}\end{array}$ & grande demônio & kidiabo \\
\hline Mwene kikadiyaphemba & Grande demônio. & \\
\hline $\begin{array}{l}\text { kimutu }[\mathrm{ki}+\mathrm{mu}+\mathrm{tu}] \\
\qquad[\mathrm{PA} 7+\mathrm{PN} 1+\mathrm{TN}]\end{array}$ & senhorzão, má pessoa, homem forte, etc. & kipessoa \\
\hline Wakexile kimutu & $\begin{array}{l}\text { Era um homem forte/ uma grande pessoa/ } \\
\text { uma má pessoa. }\end{array}$ & \\
\hline
\end{tabular}

Tabela 7: Estrutura do aumentativo em quimbundo, em português padrão e em português de Cazengo

Fonte: produzida pelos autores

Como pudemos observar, em quimbundo, o aumentativo e o diminutivo concretizam-se pela anteposição de morfemas modificadores aos temas nominais (ou radicais). $\mathrm{O}$ aumentativo é expresso pelo prefixo ki, de classe 7 , enquanto o diminutivo se concretiza no prefixo ka, de classe 12, para o singular, e no prefixo tu, de classe 13, para o plural.

\section{CONSIDERAÇÕES FINAIS}

Os exemplos aqui analisados provêm de um corpus de produções espontâneas recolhido pelos autores na região Kwanza Norte, em Angola, tendo sido analisados a partir da qualidade de falante nativo de quimbundo de um dos autores deste texto. Analisamos a variação de três estruturas morfossintáticas específicas: a) formação do plural; b) concordância de gênero e c) formação do diminutivo e aumentativo dos nomes.

Para além das interferências do quimbundo no português falado no município de Cazengo, foi possível evidenciar a existência de duas gramáticas diferenciadas, na medida em que os seus elementos gramaticais não convergem.

Ao fazer alusão a fatos do passado da comunidade estudada, por meio de dados sobre a civilização do município, este estudo permitiu-nos, igualmente, contribuir para uma pequena parcela da história da variedade angolana do português. Todavia, algumas das questões apresentadas dizem respeito a questões de política linguística em Angola, principalmente no que tange à legitimação de um português angolano. Alguns dados aqui comentados poderão levar a que os habitantes de Cazengo e de outras comunidades vizinhas compreendam que a convivência de línguas diferentes, num mesmo espaço geográfico, pode desencadear em situações de interferências linguísticas, mas que isso não se constitui num obstáculo ao aprendizado das duas línguas em questão. O nosso conhecimento dessa realidade indica-nos que a língua portuguesa é o idioma que os habitantes de Cazengo mais dominam, sendo que a supremacia do português sobre o quimbundo é fruto de políticas do Estado angolano em relação à língua portuguesa, sobretudo o fato de esta, além de língua veicular, ser a língua de escolarização. 


\section{REFERÊNCIAS}

ALMEIDA, J. A transferência linguística e a tradução: barreira à tradução ou eficaz solução comunicativa? Porto: Faculdade de Letras, 2001.

MINGAS, A. Interferência do quimbundo no português falado em Lwanda. Porto: Campo das Letras, 2000.

NGUNGA, A. Interferência de Línguas Moçambicanas em Português falado em Moçambique. Revista Científica da Universidade Eduardo Mondlane, Série: Letras e Ciências Sociais, v. 1, p. 7-12, 2012.

PERES, D. Apontamento de morfologia das línguas nacionais africanas. Luanda: Universidade Agostinho Neto, 2006.

RAPOSO, E. et al. (Org.). Gramática do português. Volume I. Lisboa: Fundação Calouste Gulbenkian, 2013.

SANTANA, M. O sufixo diminutivo em português: funcionamento e significação - do século XIII ao XX. São Paulo: Universidade S. Paulo, Faculdade de Filosofia, Letras e Ciências Humanas, 2017.

SASSOMA, J. Interferência da língua umbundu na língua portuguesa na região de Benguela. Benguela: Universidade Buila Katyavala, 2015.

\section{() (1) $\circledast \oplus$}

